\title{
Characterization by Suppression Subtractive Hybridization of Transcripts That Are Differentially Expressed in Leaves of Anthracnose-Resistant Ramie Cultivar
}

\author{
Wang Xuxia • Chen Jie • Wang Bo • Liu Lijun • \\ Jiang Hui • Tang Diluo • Peng Dingxiang
}

Published online: 27 September 2011

(C) The Author(s) 2011. This article is published with open access at Springerlink.com

\begin{abstract}
For the purpose of screening putative anthracnose resistance-related genes of ramie (Boehmeria nivea L. Gaud), a cDNA library was constructed by suppression subtractive hybridization using anthracnose-resistant cultivar Huazhu no. 4. The cDNAs from Huazhu no. 4, which were infected with Colletotrichum gloeosporioides, were used as the tester and cDNAs from uninfected Huazhu no. 4 as the driver. Sequencing analysis and homology searching showed that these clones represented 132 single genes, which were assigned to functional categories, including 14 putative cellular functions, according to categories established for Arabidopsis. These 132 genes included 35 disease resistance and stress tolerance-related genes including putative heatshock protein 90, metallothionein, PR-1.2 protein, catalase gene, WRKY family genes, and proteinase inhibitor-like protein. Partial disease-related genes were further analyzed by reverse transcription PCR and RNA gel blot. These expressed sequence tags are the first anthracnose resistancerelated expressed sequence tags reported in ramie.
\end{abstract}

Keywords Boehmeria nivea L. Gaud - Anthracnose · Pathogenesis-related genes · Suppression subtractive hybridization $(\mathrm{SSH})$

\section{Introduction}

Ramie (Boehmeria nivea L. Gaud), a perennial herbaceous plant grown for its fibers and a member of the Urticaceae

W. Xuxia $\cdot$ C. Jie $\cdot$ W. Bo $(\bowtie) \cdot$ L. Lijun $\cdot$ J. Hui $\cdot$ T. Diluo P. Dingxiang

College of Plant Science and Technology,

Huazhong Agricultural University,

Wuhan 430070 Hubei Province, People's Republic of China

e-mail: wangbo0550@yahoo.com.cn family, is planted principally in China and other Asian countries including the Philippines, India, South Korea, and Thailand (Wang et al. 2008). Ramie fibers are long and strong and possess good durability and absorbency with excellent luster (Zheng et al. 2000). In China, about 130,000 ha are planted with ramie. Ramie crops are usually infected by diseases caused by fungi, viruses, or nematodes, which decrease the yield as well as the quality of the fibers. Anthracnose is a major disease of ramie caused by the fungus Colletotrichum, first described in 1831 by Corda. Its classification is phylum Deuteromycotina, class Coelomycetes, order Melanconiales, family Melanconiaceae. This important plant fungal disease is widespread in temperate zones, particularly in subtropical and tropical regions. It often damages fruit trees, vegetables, flowers, medicinal plants, and the fruit, stems, and leaves of field crops (Sutton 1992; Agostini et al. 1992), causing large losses in agricultural production. In China, Colletotrichum boehmeriae Sawada has been reported as a pathogen of ramie, but with no further investigations (Sawada 1914, 1919). In our former research, we have identified the disease caused by Colletotrichum gloeosporioides (Wang et al. 2010a, b) which can infect the stalk and foliage, thereby degrading not only the quantity but also the quality of the fiber. Generally, the disease causes yield losses of $20-40 \%$ and as high as 55\% (Li and Ma 1993).

To study resistance genes in ramie, we constructed a suppression subtractive hybridization (SSH) (Diatchenko et al. 1996) library induced by C. gloeosporioides. The SSH technique enables specific cloning of expressed sequence tags (ESTs) representing genes that are differentially expressed in different mRNA populations and isolates genes without prior knowledge of their sequence or identity. It uses common molecular biological techniques that do not require specialized equipment or analyses 
(Moody 2001). This method was very useful in screening new genes and construction of cDNA library (Lin et al. 2010; Wang et al. 2011; Dipnarayan et al. 2011; Zhang et al. 2011a, b; Zhou et al. 2011; Prabu et al. 2011; Yang et al. 2011). Given the current understanding of ramie at the molecular level, this technique is a suitable method for the detection of low-abundance, differentially expressed transcripts that can lead to the isolation of putative disease resistance and defense-related genes.

\section{Materials and Methods}

\section{Material Preparation and Isolation of Colletotrichum}

Huazhu no. 4, an elite ramie cultivar in Hubei Province, China that shows high resistance to ramie anthracnose $(C$. gloeosporioides), was planted in the Ramie Germplasm Resources Garden of Huazhong Agricultural University. The plants used in this study were transplanted into the greenhouse under daylight with additional illumination provided by high-pressure sodium lamps to give a photoperiod of $12 \mathrm{~h}$.

C. gloeosporioides samples were collected from anthracnose lesions on ramie, the Bast Fiber Crops Institute in Changsha, Hunan Province, China. After surface sterilization of lesions with $0.1 \%$ mercuric chloride for $40 \mathrm{~s}$, followed by autoclaved water wash three times, small blocks $\left(9 \mathrm{~mm}^{2}\right)$ of diseased bark were aseptically transferred to potato dextrose agar (PDA) plates. Cultures were dark-cultured at $25^{\circ} \mathrm{C}$ incubator. C. gloeosporioides isolates were purified by single spore culturing (Choi et al. 1999). Spore masses were picked up with a sterilized wire loop and streaked on to the surface of water agar plates which were dark-cultured at $25^{\circ} \mathrm{C}$ incubator overnight. A single germinated spore was picked up with a sterilized needle and then transferred onto PDA plates. Pure cultures were stored at $4^{\circ} \mathrm{C}$ on PDA slants. Isolates were deposited in Huazhong Agricultural University, Hubei Province, China.

The ramie Huazhu no. 4 was sprayed with $20 \mathrm{ml}$ of a solution of anthracnose spores $\left(1 \times 10^{6} / \mathrm{ml}\right)$. The infected ramie plants and the control samples were cultured under conditions of $25^{\circ} \mathrm{C}$ and $80 \%$ humidity. Leaves were taken $6,12,24,36,48$, and $72 \mathrm{~h}$ after inoculation. The samples were then quick-frozen in liquid nitrogen and stored at $-80^{\circ} \mathrm{C}$ for RNA extraction.

\section{Total RNA and mRNA Isolation}

Total RNA was extracted from the samples by an optimized method (Wang et al. 2010a, b). The extracted total RNA was checked by electrophoresis on $1 \%$ agarose gels. mRNA was isolated using a PolyATract mRNA Isolation System kit (Promega, USA) according to the manufacturer's instructions.

\section{SSH Library Construction}

An SSH library was constructed using a Clontech PCRSelectTM cDNA Subtraction Kit (BD Biosciences, USA) based on the manufacturers' instructions. The final PCR products were purified using an SBS Quick PCR Purification kit (SBS Genetech, Wuhan, China). The subtracted cDNA was inserted directly into pGEM $^{\circledR}-\mathrm{T}$ Easy Vector (Promega, USA) and then transformed into JM-109 Escherichia coli cells (Promega, USA) plated onto lysogeny broth (LB) agar containing $100 \mathrm{mg} \mathrm{L}^{-1}$ ampicillin, $1 \mathrm{mM}$ isopropyl $\beta$-D-thiogalactopyranoside, and $80 \mathrm{mg} \mathrm{L}^{-1}$ 5-bromo-4-chloro-3-indolyl $\beta$-D-galactopyranoside. Following incubation at $37^{\circ} \mathrm{C}$ overnight, positive transformants based on blue/white color selection were picked and arrayed into 96-well microplates and then cultured in LB medium containing $100 \mathrm{mg} \mathrm{L}^{-1}$ ampicillin. The resultant subtractive cDNA library was stored at $-80^{\circ} \mathrm{C}$ with $15 \%$ glycerol.

\section{Amplification of cDNA Inserts}

The cDNA inserts were amplified by PCR in a 96-well plate (PTC-100, Germany) with nested PCR primer 1 and primer $2 \mathrm{R}$, which were included in the PCRSelectTM cDNA Subtraction Kit. PCR reactions $(25 \mu \mathrm{L})$ contained $17.3 \mu \mathrm{L}$ distilled water, $1 \mu \mathrm{L}$ bacterial cultures, $1 \mu \mathrm{L}$ each of nested PCR primer 1 and primer $2 \mathrm{R}(10 \mu \mathrm{M}$ each), $2.5 \mu \mathrm{L} 10 \times$ ExTaq buffer, $1.5 \mu \mathrm{L} \mathrm{Mg}{ }^{2+}$ buffer $(25 \mathrm{mM}), 0.5 \mu \mathrm{L}$ dNTPs $(10 \mathrm{mM}$ each), and $1 \mathrm{U}$ ExTaq polymerase (SBS Genetech, Wuhan, China). Reaction samples were first denatured at $94^{\circ} \mathrm{C}$ for $5 \mathrm{~min}$, followed by 30 cycles at $94^{\circ} \mathrm{C}$ for $20 \mathrm{~s}, 55^{\circ} \mathrm{C}$ for $45 \mathrm{~s}$, and $72^{\circ} \mathrm{C}$ for $45 \mathrm{~s}$, with a final extension at $72^{\circ} \mathrm{C}$ for $10 \mathrm{~min}$. All PCR products were analyzed by agarose gel electrophoresis and clones of segments $<200 \mathrm{bp}$ or two or more segments eliminated.

\section{Preparation of Probes and Differential Screening} of the Subtracted Libraries

To exclude false-positive clones and to provide further data on the relative expression levels of the cloned cDNAs, initial screening of the library to remove false positives was performed by reverse northern analysis with total labeled cDNA from unsubtracted tester cDNA and unsubtracted driver cDNA as probes. Total RNAs were extracted from healthy ramie leaves and inoculated leaves. Then, they were reversed into cDNA separately. And then, they were labeled as tester cDNA and driver cDNA probes. 
Five microliters of PCR products (about $100 \mathrm{ng}$ ) was mixed with $5 \mu \mathrm{L}$ fresh $0.6 \mathrm{M} \mathrm{NaOH}$ for denaturation and $1.2 \mu \mathrm{L}$ of the mixture was printed onto two Hybond-N+ nylon membranes (Boehringer, Mannheim, Germany). The membranes were neutralized in $0.5 \mathrm{M}$ Tris- $\mathrm{HCl}(\mathrm{pH} 7.5)$ for $5 \mathrm{~min}$ and rinsed in distilled water for $30 \mathrm{~s}$. Samples were cross-linked to the membranes by baking for $2 \mathrm{~h}$ at $80^{\circ} \mathrm{C}$ and then stored at $-4^{\circ} \mathrm{C}$ until use. Unsubtracted driver cDNA and tester cDNA probes were labeled using a DIG High Prime DNA Labeling and Detection Starter Kit II (Roche Diagnostics GmbH, Germany). Preparation of probes and hybridization were performed exactly according to the manufacturer's instructions. The results of two hybridizations were recorded for each clone, and those showing the most marked differential expression were selected for sequencing (Sunny Biotechnology, Shanghai, China).

\section{EST Sequencing and Analysis}

Homology searches were conducted for all sequences using the GenBank database and the BLAST algorithms at the National Center for Biotechnology Information (NCBI) network service (http://www.ncbi.nlm.nih.gov/BLAST/). The cDNAs were classified according to the $E$-values generated in the BLAST searches. $E$-values $<1 \mathrm{e}-10$ were deemed to indicate significant homology and functional assignment. ESTs with E-values $>1 \mathrm{e}-10$ were deemed to have no significant homology to any known protein and assumed to be novel. EST sequences were assigned manually to functional categories based on a previous catalog system (Bevan et al. 1998).

\section{RT-PCR Analysis}

Total RNA was extracted from samples taken 6, 12, 24, 36, 48 and $72 \mathrm{~h}$ after inoculation. First-strand cDNAs were generated from 50 to $100 \mathrm{ng}$ RNA samples using a reverse transcription PCR (RT-PCR) kit (Toyobo, Japan). To determine the expression of candidate genes, PCR was performed with one tenth of the first-strand cDNA template and the gene-specific primer pairs. Gene-specific RT-PCR primers were designed with Primer Premier 5.0 according to the cDNA sequences and were synthesized commercially (SBS Genetech, Wuhan, China). The following primer pairs were used: for biquitin-conjugating enzyme, fwd (5'AAGCGACGATAATGGGTC-3') and rev (5'-GCTATT GATGTTCGGGTGA-3'); for polypeptide for cytokininrepressed mRNA, fwd (5'-AACACTATCGTCGGAGG-3') and rev (5'-CAAGAAGGCACAGAGCAG-3'); for putative heat-shock protein 90, fwd (5'-AAGACCCTTAAACAA CAAGA-3') and rev (5'-AGTCGAGCCTAAACATCAG-3'); for actin factor, fwd (5'-GCCTGCTGCTTCCATTCC-3') and rev (5'-TGGCTTACATTGCCCTTGA-3'); for pathogenesisrelated protein, fwd (5'-CGGGCAACTACAATGGAGAA$\left.3^{\prime}\right)$ and rev (5'-AGCCATTTCAGAATCAAC-3'); for 3hydroxyacyl-CoA dehydrogenase, fwd (5'-ATCCGTAACA GACCAAGA-3') and rev (5'-GAGACAAACATCG CAAGT-3'); for Glycine max nodulin-26 mRNA, fwd (5'GAGAAATAGCAAACCAACCC-3') and rev (5'-CCCAC CACGGACTACTGA-3'); for pathogenesis-related protein PR10, fwd (5'-GAAGGGCGGGCATGAGAT-3') and rev (5'-TGTCGCTTTGTTTAGTTGTAGG-3'); and for stress and pathogenesis-related protein, fwd (5'-GGCATGAGAT CAAGGAGG-3') and rev (5'-TGTCGCTTTGTTTAGTTG TAGG-3'). The programs differed because of the different primers. The PCR products $(8 \mu \mathrm{L})$ for each sample were electrophoresed in a $1.5 \%$ ethidium bromide agarose gel and viewed under ultraviolet light.

Northern Blot Analysis

Total RNA (20 $\mu \mathrm{g})$ from samples taken at different times after inoculation were separated on a $1.0 \%$ agarose/formaldehyde gel and transferred to Hybond-N+ nylon membranes, by downward capillary transfer with $20 \times$ SSC. Specific probes were generated by purified PCR amplification of the relevant differentially expressed clones and labeled using a DIG High Prime DNA Labeling and Detection Starter Kit II (Roche Diagnostics GmbH, Germany). Labeling of probes and hybridization were performed exactly according to the manufacturer's instructions.

\section{Results and Discussion}

A subtracted cDNA library was constructed using the healthy ramie Huazhu no. 4 cultivar and Huazhu no. 4 inoculated with $C$. gloeosporioides. The library comprised approximately 1,000 clones, with insertions ranging from 50 to $1,100 \mathrm{bp}$. One hundred seventy-two clones of greater signal intensity were found to be regulated and were sequenced. The EST sequences obtained were identified by homology searches in BLASTN and BLASTX databases. The partial results are summarized in Table 1. One hundred thirty-two single ESTs were assigned to functional categories and classified into 14 putative cellular functions, basing on the functional categories established for Arabidopsis (Bevan et al. 1998). The distribution of the genes is illustrated in Fig. 1. In the 132 single ESTs, 70 had high similarity to database entries. The largest set of genes (28\%) was assigned to disease/defense. Unclear classification and unclassified were the second $(17 \%)$ and the third (11\%) largest groups, respectively. Based on the results of northern hybridization and EST sequencing, RT-PCR was 
Table 1 Representative clones of SSH library from Boehmeria infected by C. gloeosporioides

\begin{tabular}{|c|c|c|c|c|}
\hline $\begin{array}{l}\text { Clone } \\
\text { no. }\end{array}$ & $\begin{array}{l}\text { Number of identical } \\
\text { clones }^{\mathrm{a}}\end{array}$ & $\begin{array}{l}\text { Best } \\
E \text {-value }\end{array}$ & $\begin{array}{l}\text { Identity } \\
\text { percentage }(\%)\end{array}$ & Best match in database \\
\hline \multicolumn{5}{|c|}{ Pathogenesis-related proteins/plant defense } \\
\hline 15 & emb|CT029927.1| & $8 \mathrm{e}-10$ & 92 & Poplar cDNA sequences (SSH library infected with rust fungus) \\
\hline 39 & gb|AAT02522.1| & 0.006 & 62 & Metallothionein 1a \\
\hline 42 & $\mathrm{gb}|\mathrm{DQ} 907240.1|$ & 0.059 & 90 & Heat-shock transcription factor 1 \\
\hline 46 & emb|CT029469.1| & $8 \mathrm{e}-10$ & 92 & Poplar cDNA sequences (SSH library infected with rust fungus) \\
\hline 72 & ref|XM_002519612.1| & $8 e^{-95}$ & 83 & (S)-2-hydroxy-acid oxidase \\
\hline 73 & $\mathrm{emb}|\mathrm{CT} 029702.1|$ & 0.063 & 100 & Poplar cDNA sequences (SSH library infected with rust fungus) \\
\hline 88 & $\mathrm{gb}|\mathrm{EU} 311423.1|$ & $3 e-21$ & 77 & Heat-shock protein 90 mRNA \\
\hline $141-1$ & ref|XM_002273749.1| & $2 \mathrm{e}-09$ & 75 & Thaumatin-like protein \\
\hline 185 & $\mathrm{gb}|\mathrm{AY} 919870.1|$ & $4 e^{-21}$ & 70 & Betula platyphylla metallothionein-like protein mRNA \\
\hline 196 & $\mathrm{emb}|\mathrm{CU} 231781.1|$ & $6 e-63$ & 80 & Populus EST from mildly drought-stressed leaves \\
\hline 205 & $\mathrm{gb}|\mathrm{DQ} 186610.1|$ & $3 e-53$ & 90 & Catharanthus roseus calmodulin 2 mRNA \\
\hline 223 & emb|AJ007349.1|TAE7349 & 0.002 & 61 & Triticum aestivum mRNA for PR-1.2 protein \\
\hline 235 & emb|CT028832.1| & 0.019 & 100 & Poplar cDNA sequences \\
\hline 248 & emb|CAD42908.1| & $3 e-27$ & 78 & Catalase \\
\hline 367 & $\mathrm{gb}|\mathrm{EU} 586200.1|$ & $4 e^{-135}$ & 86 & Biquitin-conjugating enzyme 2 mRNA \\
\hline 372 & $\mathrm{gb} \mid$ AAL36933.1|AF213481_1 & $2 \mathrm{e}-10$ & 100 & Gamma-tocopherol methyltransferase \\
\hline 425 & emb|CT028832.1| & 0.02 & 100 & Poplar cDNA sequences (SSH library infected with rust fungus) \\
\hline 426 & $\mathrm{gb}|\mathrm{AY} 847454.1|$ & $1 \mathrm{e}-07$ & 74 & Metallothionein class II \\
\hline 459 & $\mathrm{gb}|\mathrm{EU} 019559.1|$ & $3 e-52$ & 83 & Glycine max WRKY14 \\
\hline 495 & $\mathrm{emb}|\mathrm{CU} 231781.1|$ & $1 e^{-54}$ & 76 & Populus EST from mildly drought-stressed leaves \\
\hline 578 & $\mathrm{gb}|\mathrm{EU} 024476.1|$ & $1 \mathrm{e}-07$ & 97 & Arachis hypogaea oxalate oxidase mRNA \\
\hline 585 & $\mathrm{gb}|\mathrm{DQ} 497797.1|$ & $8 \mathrm{e}-40$ & 74 & Lipoxygenase 2 \\
\hline 589 & emb|AJ007349.1| & $4 e^{-04}$ & 86 & Triticum aestivum mRNA for PR-1.2 protein \\
\hline 599 & dbj|AB091685.1| & $3 e-04$ & 100 & Daidzein 7-0-methyltransferase \\
\hline $661-2$ & emb|AM748435.1| & $5 e-07$ & 84 & $\begin{array}{l}\text { Vigna unguiculata partial mRNA for putative plant disease } \\
\text { resistance response protein family }\end{array}$ \\
\hline 640 & ref|XM_002519612.1| & $7 e^{-95}$ & 84 & Ricinus communis (S)-2-hydroxy-acid oxidase \\
\hline 667 & $\mathrm{gb}|\mathrm{AY} 594295.1|$ & $8 \mathrm{e}-17$ & 68 & Populus trichocarpa $\times$ Populus deltoides metallothionein 1a (MT1a) \\
\hline 696 & $\mathrm{gb}|\mathrm{EU} 117126.1|$ & $4 e^{-24}$ & 81 & $\begin{array}{l}\text { Prunus domestica cultivar Violette pathogenesis-related } \\
\text { protein PR10 }\end{array}$ \\
\hline 702 & $\mathrm{emb}|\mathrm{CU} 232440.1|$ & $1 e^{-07}$ & 91 & Populus EST from mildly drought-stressed leaves \\
\hline 704 & emb|AM748435.1| & $5 e-12$ & 91 & Putative plant disease resistance response protein family \\
\hline 719 & emb|AJ130889.1|FSY130889 & $6 e-14$ & 81 & Fagus sylvatica mRNA for stress and pathogenesis-related protein \\
\hline 761 & $\mathrm{gb}|\mathrm{EU} 526202.1|$ & $8 e^{-13}$ & 73 & PR10 protein \\
\hline 766 & $\mathrm{gb}|\mathrm{AY} 158153.1|$ & $4 \mathrm{e}-09$ & 92 & Citrus $\times$ paradisi type I proteinase inhibitor \\
\hline 792 & emb|AJ496418.1|PPE496418 & $7 e-42$ & 79 & Prunus persica mRNA for catalase \\
\hline 933 & $\mathrm{emb}|\mathrm{CU} 226741.1|$ & $2 \mathrm{e}-89$ & 83 & Populus EST from severely drought-stressed leaves \\
\hline \multicolumn{5}{|c|}{ Metabolism } \\
\hline 96 & $\mathrm{gb}|\mathrm{EF} 568776.1|$ & $7 e-109$ & 81 & Prunus persica beta-galactosidase protein \\
\hline 288 & $\mathrm{gb}|\mathrm{EU} 106889.1|$ & $1 \mathrm{e}^{-14}$ & 79 & Jatropha curcas chloroplast omega- 6 fatty acid desaturase mRNA \\
\hline 600 & ref|XM_002514233.1| & $2 \mathrm{e}-51$ & 77 & Ricinus communis 3-hydroxyacyl-CoA dehydrogenase \\
\hline 664 & $\mathrm{dbj}|\mathrm{AB} 221013.2|$ & $5 e^{-42}$ & 83 & Beta vulgaris BvSDC1 mRNA for putative serine decarboxylase \\
\hline 707 & ref|XM_002283715.1| & $2 \mathrm{e}-25$ & 85 & Vitis vinifera malate dehydrogenase $\left(\mathrm{NADP}^{+}\right)$ \\
\hline 740 & ref|XM_002515921.1| & $2 \mathrm{e}-53$ & 85 & Ricinus communis malic enzyme \\
\hline \multicolumn{5}{|c|}{ Cell growth/division } \\
\hline 178 & $\mathrm{gb}|\mathrm{FJ} 226587.1|$ & $4 e^{-76}$ & 80 & Glycine max late-embryogenesis abundant protein 2 \\
\hline 233 & $\mathrm{gb}|\mathrm{FJ} 156098.1|$ & $4 e^{-43}$ & 80 & Translationally controlled tumor protein \\
\hline
\end{tabular}


Table 1 (continued)

\begin{tabular}{|c|c|c|c|c|}
\hline $\begin{array}{l}\text { Clone } \\
\text { no. }\end{array}$ & $\begin{array}{l}\text { Number of identical } \\
\text { clones }^{\mathrm{a}}\end{array}$ & $\begin{array}{l}\text { Best } \\
E \text {-value }\end{array}$ & $\begin{array}{l}\text { Identity } \\
\text { percentage }(\%)\end{array}$ & Best match in database \\
\hline 587 & ref|XM_002516884.1| & 0.012 & 87 & Translationally controlled tumor protein \\
\hline 645 & dbj|D29684.1|CUSCR9 & $4 e-21$ & 83 & Polypeptide for cytokinin-repressed mRNA \\
\hline 663 & emb|AJ252088.1| & $7 e-05$ & 77 & mRNA for gibberellin 20 -oxidase \\
\hline 757 & $\mathrm{emb}|\mathrm{AJ} 238624.1|$ & $2 \mathrm{e}-89$ & 88 & Translation initiation factor \\
\hline \multicolumn{5}{|c|}{ Transporters } \\
\hline 24 & ref|XM_002336761.1| & $2 \mathrm{e}-38$ & 76 & Populus trichocarpa oligopeptide transporter OPT family \\
\hline 153 & emb|AJ534339.1| & 0.008 & 86 & $\begin{array}{l}\text { Agaricus bisporus partial mRNA for putative } \\
\text { inorganic phosphate transporter (ipt gene) }\end{array}$ \\
\hline 189 & ref|XM_002336761.1| & $8 \mathrm{e}-36$ & 75 & Populus trichocarpa oligopeptide transporter OPT family \\
\hline 457 & emb|AJ534339.1| & $3 e-04$ & 88 & mRNA for putative inorganic phosphate transporter \\
\hline $660-2$ & $\mathrm{emb}|\mathrm{AJ} 272202.1|$ & $2 \mathrm{e}-06$ & 90 & Arabidopsis thaliana mRNA for mitochondrial half-ABC transporter \\
\hline 665 & $\mathrm{gb}|\mathrm{EF} 453694.1|$ & $2 \mathrm{e}-70$ & 78 & $\begin{array}{l}\text { Populus trichocarpa Mn-specific cation diffusion facilitator } \\
\text { transporter (MTP11.2) mRNA }\end{array}$ \\
\hline 787 & $\mathrm{gb}|\mathrm{DQ} 372573.1|$ & $1 \mathrm{e}-05$ & 84 & Gossypium hirsutum transferring glycosyl protein (gt) mRNA \\
\hline \multicolumn{5}{|c|}{ Transcription } \\
\hline 218 & emb|AM293617.1| & 0.075 & 100 & Platanus $\times$ acerifolia partial mRNA for putative transcription factor \\
\hline 227 & emb|AM397241.1| & 0.006 & 93 & Ty1-copia reverse transcriptase (RT gene) and partial DfRedu pseudogene \\
\hline 230 & dbj|AB235910.1| & 0.20 & 91 & Triticum aestivum gene for RNA ligase isoform 3 \\
\hline 650 & emb|AM293617.1| & 0.002 & 100 & Platanus $\times$ acerifolia partial mRNA for putative transcription factor \\
\hline 651 & $\mathrm{gb}|\mathrm{AF} 190657.1| \mathrm{AF} 190657$ & $3 e-39$ & 83 & Nicotiana tabacum clone 7 poly(A)-binding protein (PABP) mRNA \\
\hline 926 & $\mathrm{gb}|\mathrm{AY} 224135.1|$ & $7 e-11$ & 77 & Pelargonium $\times$ hortorum 4 coumarate CoA ligase mRNA \\
\hline 929 & $\mathrm{gb}|\mathrm{AY} 224135.1|$ & $5 e-12$ & 75 & Pelargonium $\times$ hortorum 4 coumarate $\mathrm{CoA}$ ligase mRNA \\
\hline $936-1$ & $\mathrm{gb}|\mathrm{AY} 224135.1|$ & $2 \mathrm{e}-16$ & 81 & Pelargonium $\times$ hortorum 4 coumarate $\mathrm{CoA}$ ligase mRNA \\
\hline $943-1$ & $\mathrm{gb}|\mathrm{AY} 224135.1|$ & $2 \mathrm{e}-17$ & 82 & Pelargonium $\times$ hortorum 4 coumarate $\mathrm{CoA}$ ligase mRNA \\
\hline $971-1$ & $\mathrm{gb}|\mathrm{AY} 224135.1|$ & $1 \mathrm{e}-06$ & 71 & Pelargonium $\times$ hortorum 4 coumarate $\mathrm{CoA}$ ligase mRNA \\
\hline \multicolumn{5}{|c|}{ Signal transduction } \\
\hline 205 & $\mathrm{gb}|\mathrm{AF} 292108.1| \mathrm{AF} 292108$ & $3 e-97$ & 90 & Prunus avium calmodulin mRNA \\
\hline 367 & $\mathrm{gb}|\mathrm{EU} 586200.1|$ & $4 e^{-135}$ & 86 & Ubiquitin-conjugating enzyme 2 \\
\hline 449 & ref|XM_002275581.1| & $3 e-80$ & 79 & Vitis vinifera ubiquitin-activating enzyme \\
\hline 584 & ref|XM_002512452.1| & $2 e^{-73}$ & 83 & Ricinus communis GTP-binding protein sar1 \\
\hline \multicolumn{5}{|c|}{ Transposons } \\
\hline $495-2$ & $\mathrm{gb}|\mathrm{EU} 558534.1|$ & $8 \mathrm{e}-40$ & 76 & Arabidopsis lyrata clone SINE9 transposon-insertion display band \\
\hline $936-2$ & $\mathrm{gb}|\mathrm{EU} 558528.1|$ & $1 e^{-34}$ & 81 & $\begin{array}{l}\text { Arabidopsis lyrata clone CACTA2 transposon-insertion display } \\
\text { band genomic sequence }\end{array}$ \\
\hline 944 & $\mathrm{gb}|\mathrm{EU} 558534.1|$ & $4 e-25$ & 72 & $\begin{array}{l}\text { Arabidopsis lyrata clone SINE9 transposon-insertion display band } \\
\text { genomic sequence }\end{array}$ \\
\hline
\end{tabular}

\section{Energy}

118 ref|XM_002526592.1| $8 \mathrm{e}-23 \quad 70$

195 emb|AM748509.1 $\quad 0.003 \quad 100$

Protein synthesis

$\begin{array}{llll}119 & \text { ref|XM_002519706.1| } & 8 \mathrm{e}-52 & 82 \\ 194 & \text { emb|AJ251333.1| } & 4 \mathrm{e}^{-71} & 81 \\ 754 & \text { emb|AJ831469.1| } & 1 \mathrm{e}-09 & 76 \\ 756 & \text { emb|AJ697756.1| } & 0.026 & 88\end{array}$

Protein destination and storage

$446 \quad$ emb|AM748427.1|
$0.005 \quad 91$
Translationally controlled tumor protein

mRNA for gibberellin 20-oxidase

Populus trichocarpa oligopeptide transporter OPT family Agaricus bisporus partial mRNA for putative inorganic phosphate transporter (ipt gene)

Populus trichocarpa oligopeptide transporter OPT family mRNA for putative inorganic phosphate transporter

Arabidopsis thaliana mRNA for mitochondrial half-ABC transporter

Platanus $\times$ acerifolia partial mRNA for putative transcription factor Ty1-copia reverse transcriptase (RT gene) and partial DfRedu pseudogene Triticum aestivum gene for RNA ligase isoform 3

Platanus $\times$ acerifolia partial mRNA for putative transcription factor Nicotiana tabacum clone 7 poly(A)-binding protein (PABP) mRNA

Pelargonium $\times$ hortorum 4 coumarate CoA ligase mRNA

Pelargonium $\times$ hortorum 4 coumarate $\mathrm{CoA}$ ligase mRNA

Pelargonium $\times$ hortorum 4 coumarate $\mathrm{CoA}$ ligase mRNA

Pelargonium $\times$ hortorum 4 coumarate $\mathrm{CoA}$ ligase mRNA

Pelargonium $\times$ hortorum 4 coumarate CoA ligase mRNA

Prunus avium calmodulin mRNA

Ricinus communis photosystem I reaction center subunit N

Vigna unguiculata partial mRNA for putative single-stranded nucleic acid binding $\mathrm{R} 3 \mathrm{H}$

Ricinus communis $60 \mathrm{~S}$ ribosomal protein $\mathrm{L} 32$

Pisum sativum aair gene for acetohydroxy acid isomeroreductase Pisum sativum mRNA for putative glycine-rich protein precursor Eucalyptus globulus subsp. globulus partial mRNA for putative ribosomal protein

Vigna unguiculata partial mRNA for putative cathepsin B-like cysteine protease 
Table 1 (continued)

\begin{tabular}{|c|c|c|c|c|}
\hline $\begin{array}{l}\text { Clone } \\
\text { no. }\end{array}$ & $\begin{array}{l}\text { Number of identical } \\
\text { clones }^{\mathrm{a}}\end{array}$ & $\begin{array}{l}\text { Best } \\
E \text {-value }\end{array}$ & $\begin{array}{l}\text { Identity } \\
\text { percentage (\%) }\end{array}$ & Best match in database \\
\hline \multicolumn{5}{|c|}{ Intracellular traffic } \\
\hline 609 & $\mathrm{gb}|\mathrm{L} 12257.1|$ & $6 e^{-71}$ & 82 & Glycine max nodulin-26 mRNA \\
\hline 654 & $\mathrm{gb}|\mathrm{GQ} 354526.1|$ & 0.002 & 91 & Dekkera bruxellensis strain CBS 2499 mitochondrion \\
\hline 736 & $\mathrm{gb}|\mathrm{L} 12257.1|$ & $7 e^{-71}$ & 82 & Glycine max nodulin-26 mRNA \\
\hline 745 & ref|XM_002522770.1| & $2 \mathrm{e}-18$ & 74 & Ricinus communis mitochondrial ribosomal protein \\
\hline \multicolumn{5}{|c|}{ Cell structure } \\
\hline 204 & $\mathrm{gb}|\mathrm{AY} 487315.1|$ & $3 e-97$ & 89 & Ficus carica actin 1 mRNA \\
\hline $943-2$ & emb|AJ420893.1| & $4 e^{-}-34$ & 76 & Entandrophragma cylindricum microsatellite DNA \\
\hline \multicolumn{5}{|c|}{ Secondary metabolism } \\
\hline $971-2$ & dbj|AB261987.1| & $1 \mathrm{e}-04$ & 79 & Photinus pyralis mRNA for luciferase \\
\hline
\end{tabular}

The GenBank accession number of the clones in the Table 1: GW691509-GW691606; GO653137-GO653162

${ }^{a}$ Best match in the GenBank (gb), DNA Data Bank of Japan (dbj), European Molecular Biology Laboratory (emb), or NCBI database. The accession number of the match is in parentheses

${ }^{\mathrm{b}}$ The expected $(E)$ value refers to the number of matches expected by chance alone. The lower the $E$ value, the more strongly supported the match

performed to evaluate the differential expression of selected disease-related genes (Fig. 2). As indicated by the SSH results (Table 1), we selected nine clones: ubiquitinconjugating enzyme 2 mRNA, polypeptide for cytokininrepressed, putative heat-shock protein 90 (Hsp90), actin factor, pathogenesis-related protein, 3-hydroxyacyl-CoA dehydrogenase, nodulin-26, pathogenesis-related protein PR10, and pathogenesis-related protein, all of which exhibited high homology in the BLASTX comparison with the NCBI database. RT-PCR showed high levels of the genes encoding stress and pathogenesis-related protein at the time of induction of pathology and in the control group (CK) (Fig. 2). The clone related to actin factor showed peak expression after $24 \mathrm{~h}$. Expression of some genes was induced after the induction of pathology: polypeptide for cytokinin-repressed mRNA showed differences after $12 \mathrm{~h}$ and peaked at $24 \mathrm{~h}$, after which expression was maintained. However, another clone related to the stress and pathogenesisrelated protein was expressed only at $12-48 \mathrm{~h}$. We conclude that expression of this gene was brought about the induction of pathology. The clone with high homology to putative heat-shock protein 90 was highly expressed in CK but showed a downward trend after the induction of pathology. The Hsp90 proteins might play a role either in stabilization of proteins involved in disease resistance or in assembly of multi-subunit complexes required for activation of $\mathrm{R}$ proteins
Fig. 1 Functional categories of genes included in the anthracnose-resistant subtractive cDNA library, based on the classification described in Bevan et al. (1998)

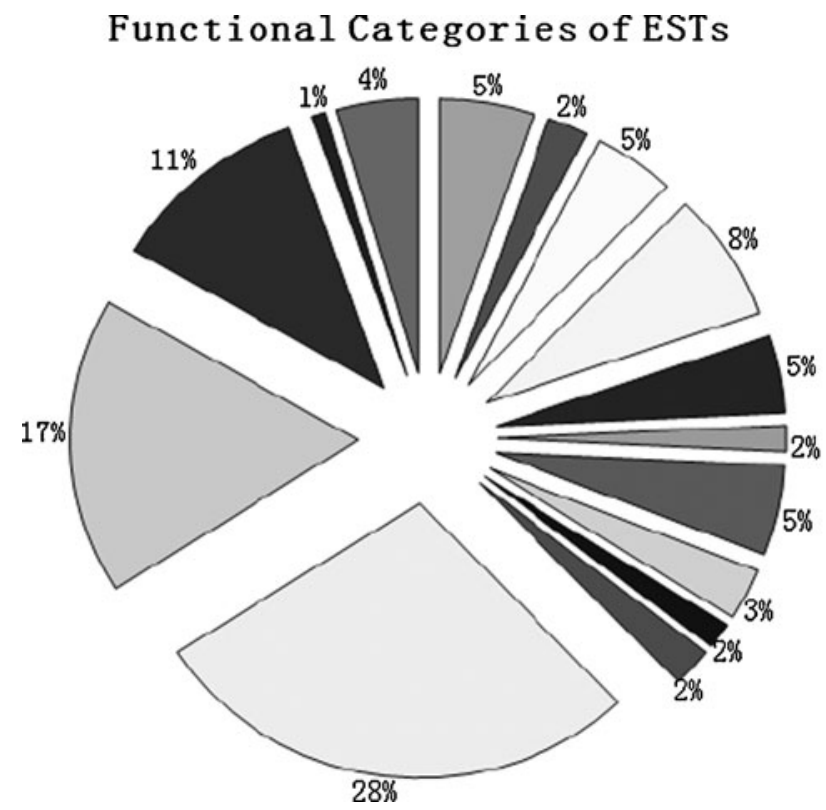

Metabolism

Energy

$\square$ Cell growth/division

$\square$ Transcription

Protein synthesis

$\checkmark$ Protein destination and storage

Transporters

$\square$ Intracellular traffic

Cell structure

Signal transcuction

$\square$ Disease/defence

Unclear classification

Unclassified

Secondary metabolism

$\square$ Transposons 
Fig. 2 RT-PCR analysis of cDNAs representative of different types of gene expression patterns. $C K$, control leaves; $6 h, 12 h, 24 h, 36 h, 48 h, 72 h, 6$, $12,24,36$, and $72 \mathrm{~h}$, respectively, after inoculation with C. gloeosporioides

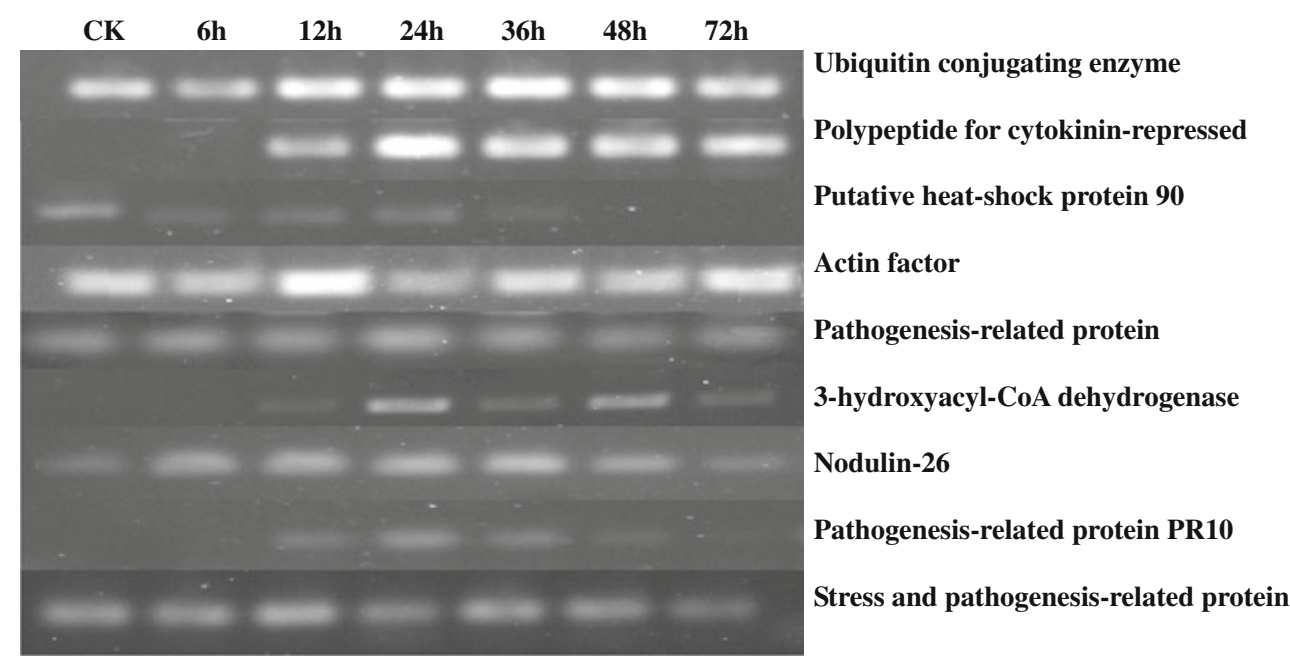

and intracellular disease resistance signaling pathways (Scofield et al. 2005). In the RNA gel blot analysis, we used probes synthesized from clone 645 (polypeptide for cytokinin-repressed) and clone 696 (pathogenesis-related protein PR10). From Fig. 3, it can be seen that it is likely that the corresponding genes were expressed at similar levels as observed by RT-PCR.

Breeding for stable anthracnose resistance is difficult because of the variation among $C$. gloeosporioides for pathogenicity and virulence. In addition, the hostpathogen interaction is poorly understood. Few reports on anthracnose and the cloning and characterization of ramie resistance gene homologs have been published. In this study, the transcription profiles of uninfected and infected leaves of the anthracnose-resistant Huazhu no. 4 ramie cultivar were compared by SSH analysis. The SSH library was not very extensive, which might be due to the efficiency of the adapters ligation, which plays a key role in the subtractive library construction. Additionally, the efficiency depends upon several factors such as the quality of cDNA synthesis, the completeness of enzyme digestion, and the quality of purification. However, we obtained many disease resistance- and defense-related ESTs. There were only about 200 ESTs of ramie in the

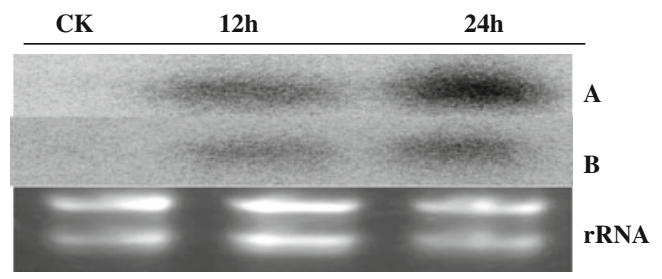

Fig. 3 Northern hybridization of total RNA from anthracnoseresistant ramie cultivar Huazhu no. 4 using clones 645 and 696. $A, B$, ECL detection of the 645 and 696 probes, separately; $C K$, control leaves; $12 \mathrm{~h}, 24 \mathrm{~h}, 12$ and $24 \mathrm{~h}$ after inoculation with $C$. gloeosporioides
GenBank database, so our results are meaningful for future research into disease resistance in ramie. We found several transcripts encoding proteins belonging to groups including pathogenesis-related proteins, metallothioneins, severe drought-stressed proteins, Hsp90, thaumatin-like protein, catalase (CAT1), the WRKY family, and oxalate oxidase.

Although PCR-selective cDNA subtraction is a powerful tool for identifying differentially expressed genes, and subtractive products may contain cDNAs that are common to or have similar levels in the two tissues, false-positive clones may occur (Zeng et al. 2006). As a result, we performed RT-PCR for further identification. According to the RT-PCR results, the resistant ramie cultivar had many resistance genes before being infected, including ubiquitinconjugating enzyme, PR-1.2 protein, nodulin-26 mRNA, and mRNAs for stress and pathogenesis-related protein. These proteins maintained their high levels before and after inoculation and played an important role in the resistance process. The pathogenesis-related proteins included several proteins that are involved in the detoxification of reactive oxygen species (Mishra et al. 2007). Another aspect of our findings was the decline of Hsp90, a molecular chaperone responsible for the folding and functions of important cellular proteins including steroid hormone receptors, protein kinases, and proteins controlling the cell cycle and apoptosis (Schulte et al. 1998). Wicklow et al. (2009) demonstrated that monorden from Colletotrichum graminicola is a potent selective inhibitor of Hsp90. Monorden might be a competitive ligand for the ATP-binding site of Hsp90, thus preventing the ATPase activity necessary for its role as a chaperone (Roe et al. 1999). It could be confirmed by further experiments on our pathogen, C. gloeosporioides. 3Hydroxyacyl-CoA dehydrogenase (HADH) deficiency is an autosomal recessive metabolic disorder, resulting from mutations in the HADH gene in chromosome 4q22-q26 
(http://www.ncbi.nlm.nih.gov/entrez/dispomim.cgi? $\mathrm{id}=231530$; Vredendaal et al. 1998), which has so far only been described in a few patients (Martins et al. 2011). In plant of castor bean (Ricinus communis) endosperm and potato tubers, this enzyme had a high expression level (Courtois-verniquet and Douce 1993).

The conclusion of this study is that, in addition to the expression of several well-characterized pathogenesisrelated proteins, high-level constitutive expression of genes encoding thaumatin-like protein, calmodulin, catalase, and proteinase inhibitor seems to be a major factor in rendering ramie resistant to infection by the fungus $C$. gloeosporioides. The recovery of many ESTs indicates that ramie quickly responds to $C$. gloeosporioides challenge and suggests that early transcriptional events can play an important role in determining whether ramie succumbs to or resists infection. This study is the first global analysis of genes in ramie with anthracnose. The ESTs identified following C. gloeosporioides challenge in this study should provide a useful genomic resource for biologists and plant breeders in developing new strategies for improving resistance to anthracnose in ramie.

Open Access This article is distributed under the terms of the Creative Commons Attribution Noncommercial License which permits any noncommercial use, distribution, and reproduction in any medium, provided the original author(s) and source are credited.

\section{References}

Agostini JP, Timmer LW, Mitchell DJ (1992) Morphological and pathological characteristic of strains of Colletotrichum gloeosporiodes from citrus. Phytopathology 82:1377-1382

Bevan $\mathrm{M}$ et al (1998) Analysis of $1.9 \mathrm{Mb}$ of contiguous sequence from chromosome 4 of Arabidopsis thaliana. Nature 391:485488

Choi YW, Hyde KD, Ho WH (1999) Single spore isolation of fungi. Fungal Divers 3:29-38

Courtois-verniquet F, Douce R (1993) Lack of aconitase in glyoxysomes and peroxisomes. Biochem J 294:103-107

Diatchenko L, Lau YFC, Campbell AP, Chenchik A, Moqadam F, Huang B, Lukyanov S, Lukyanov K, Gurskaya N, Sverdlov ED, Siebert PD (1996) Suppression subtractive hybridization: a method for generating differentially regulated or tissue-specific cDNA probes and libraries. Proc Natl Acad Sci USA 93:60256030

Dipnarayan S, Vajinder K, Shripad RB, Ramamurthy S (2011) Characterization of upstream sequences of the $L O J$ gene leads to identification of a novel enhancer element conferring lateral organ junction-specific expression in Arabidopsis thaliana. Plant Mol Biol Rep 29:265-277

Li RM, Ma HG (1993) Studies on the occurrence and control of ramie anthracnose. J Plant Prot 20(1):83-89 (In Chinese)

Lin KH, Lin CH, Chan MT, Lo HF (2010) Identification of floodingresponse genes in eggplant roots by suppression subtractive hybridization. Plant Mol Biol Rep 28:212-221
Martins E, Cardoso ML, Rodrigues E, Barbot C, Ramos A, Bennett MJ, Teles EL, Vilarinho L (2011) Short-chain 3-hydroxyacylCoA dehydrogenase deficiency: the clinical relevance of an early diagnosis and report of four new cases. J Inherit Metab Dis 34:835-842

Mishra RN, Reddy PS, Nair S, Markandeya G, Reddy AR, Sopory SK, Reddy MK (2007) Isolation and characterization of expressed sequence tags (ESTs) from subtracted cDNA libraries of Pennisetum glaucum seedlings. Plant Mol Biol 64:713-732

Moody DE (2001) Genomics techniques: an overview of methods for the study of gene expression. J Anim Sci 79:E128-E135

Prabu G, Kawar PG, Pagariya MC, Prasad DT (2011) Identification of water deficit stress upregulated genes in sugarcane. Plant Mol Biol Rep 29:291-304

Roe SM, Prodromou C, O’Brien R, Ladbury JE, Piper PW, Pearl LH (1999) Structural basis for inhibition of the Hsp90 molecular chaperone by the antitumor antibiotics radicicol and geldanamycin. J Med Chem 42:260-266

Sawada K (1914) Colletotrichum boehmeriae In: Taiwan Hakubutsu Gakkwai Kwaiho (J Formosan Nat Hist Soc) 17(2) (In Japanese)

Sawada K (1919) Taiwan agricultural research special report 19 (Taiwan Fungi Report 1), pp 571

Schulte TW, Akinaga S, Soga S, Sullivan W, Stensgard B, Toft D, Neckers LM (1998) Antibiotic radicicol binds to the N-terminal domain of Hsp90-binding agents. Cell Stress Chaperones 3:100 108

Scofield SR, Huang L, Brandt AS (2005) Development of a virusinduced gene-silencing system for hexaploid wheat and its use in functional analysis of the Lr21-mediated leaf rust resistance pathway. Plant Physiol 138(4):2165-2173

Sutton BC (1992) The genus Glomerella and its anamorph Colletotrichum. In: Colletotrichum-biology, pathology and control. CAB International, Wallingford, pp 1-26

Vredendaal J, van den Berg I, Stroobants A et al (1998) Structural organization of the human short-chain L-3-hydroxyacyl-CoA dehydrogenase gene. Mamm Genome 9:763-768

Wang B, Peng DX, Sun ZX, Zhang N, Gao SM (2008) In vitro plant regeneration from seedling-derived explants of ramie [Boehmeria nivea (L.) Gaud]. In Vitro Cell Dev Biol Plant 44:105-111

Wang XX, Wang B, Liu LJ, Chen J, Cui XP, Jiang H, Peng DX (2010a) First report of anthracnose caused by Colletotrichum gloeosporioides on ramie in China. Plant Dis 94:1508.1

Wang XX, Wang B, Liu LJ, Cui XP, Yang JY, Wang H, Jiang H, Luo BB, Long Z, Dou WX, Zhang N, Peng DX (2010b) Isolation of high quality RNA and construction of a suppression subtractive hybridization library from ramie (Boehmeria nivea L. Gaud.). Mol Biol Rep 37:2099-2103

Wang WK, Liu CC, Chiang TY, Chen MT, Chou CH, Yeh CH (2011) Characterization of expressed sequence tags from flower buds of alpine lilium formosanum using a subtractive cDNA library. Plant Mol Biol Rep 29:88-97

Wicklow DT, Jordan AM, Gloer JB (2009) Antifungal metabolites (monorden, monocillins I, II, III) from Colletotrichum graminicola, a systemic vascular pathogen of maize. Mycol Res 113 (12):1433-1442

Yang Z, Peng ZS, Yang H, Yang J, Wei S, Cai SH (2011) Suppression subtractive hybridization identified differentially expressed genes in pistil mutations in wheat. Plant Mol Biol Rep 29:431-439

Zeng FC, Zhang XL, Zhu LF, Tu LL, Guo XP, Nie YC (2006) Isolation and characterization of genes associated to cotton somatic embryogenesis by suppression subtractive hybridization and macroarray. Plant Mol Biol 60:167-183 
Zhang H, Hu YG, Wang CY, Ji WQ (2011a) Gene expression in wheat induced by inoculation with Puccinia striiformis west. Plant Mol Biol Rep 29:458-465

Zhang XD, Allan AC, Yi Q, Chen L, Li K, Shu Q, Su J (2011b) Differential gene expression analysis of Yunnan red pear, Pyrus Pyrifolia, during fruit skin coloration. Plant Mol Biol Rep 29:305-314
Zheng LS, Du YM, Zhang JY (2000) Biobleaching effect of xylanase preparation from an alkalophilic Bacillus on ramie fibers. Biotechnol Lett 22:1363-1367

Zhou MB, Yang P, Gao PJ, Tang DQ (2011) Identification of differentially expressed sequence tags in rapidly elongating Phyllostachys pubescens internodes by suppressive subtractive hybridization. Plant Mol Biol Rep 29:224-231 